\title{
Real-time Multi-Messenger Analysis Framework of $\mathrm{KM} 3 \mathrm{NeT}$
}

\section{William Assal, ${ }^{a}$ Damien Dornic, ${ }^{a}$ Feifei Huang, ${ }^{a},{ }^{*}$ Emmanuel Le Guirriec, ${ }^{a}$ Massimiliano Lincetto ${ }^{a, b}$ and Godefroy Vannoye ${ }^{a, c}$ on behalf of the KM3NeT Collaboration}

(a complete list of authors can be found at the end of the proceedings)

${ }^{a}$ Aix Marseille Univ, CNRS/IN2P3, CPPM, Marseille, France

${ }^{b}$ Ruhr University Bochum, Germany

${ }^{c}$ Ecole Normale Supérieure de Lyon, 15 parvis René Descartes BP 700069342 Lyon Cedex 07 France

E-mail: feifei.huang@cppm.in2p3.fr

KM3NeT is a multi-purpose cubic-kilometer neutrino observatory in construction in the Mediterranean Sea. It consists of ORCA and ARCA (for Oscillation and Astroparticle Research with Cosmics in the Abyss, respectively); currently both have a few detection lines in operation. Although having different primary goals, both detectors can be used to do neutrino astronomy over a wide energy range, from a few $\mathrm{GeV}$ to a few tens of $\mathrm{PeV}$. In view of the growing field of time-domain astronomy, it is increasingly crucial to be able to identify neutrinos in real-time. This online neutrino sample will serve to trigger neutrino alerts that will be sent to the astronomy community and to look for time/space coincidence around external electromagnetic and multi-messenger triggers. These real-time searches can significantly increase the discovery potential of transient cosmic accelerators and refine the pointing directions in the case of poorly localized triggers, such as gravitational waves. In the field of core-collapse supernovae (CCSN), the detection of the MeVscale CCSN neutrinos is crucial as an early warning. KM3NeT's digital optical modules act as good detectors for these neutrinos. This proceeding presents the status of KM3NeT's real-time multi-messenger activities, including supernova monitoring, online event reconstruction, event classification and selection, alert distribution, and the first test of the selection on data.

$37^{\text {th }}$ International Cosmic Ray Conference (ICRC 2021)

July 12th - 23rd, 2021

Online - Berlin, Germany

\footnotetext{
*Presenter
} 


\section{Introduction}

Multi-messenger neutrino astronomy is a growing field, it benefits from the properties of neutrinos being neutral, almost massless, and only weakly interacting. Neutrinos do not get deflected by magnetic fields during their intergalactic travel thus always point back to their origin. It can provide complementary information when combined with other cosmic messengers, like the traditional photons and the more recent gravitational waves. Neutrinos can be identified in real-time and refine the pointing direction for optical observatories to do quick follow-up studies of transient sources. In the field of core-collapse supernova neutrinos (CCSNs), neutrinos can act as an early warning as neutrinos arrive Earth hours before the photons.

KM3NeT [1], the cubic-kilometer neutrino observatory under construction in the Mediterranean Sea, will be able to detect neutrinos in a large energy range from $\mathrm{MeV}$-scale CCSNs to astrophysical neutrinos up to a few PeV. This proceeding describes the status of KM3NeT's realtime multi-messenger analysis framework.

The proceeding starts with an introduction to KM3NeT and its main science goals in Section 1. Section 2 describes the data flow and the main structure of the real-time framework. Section 3 presents the frameworks' first pipeline of CCSN neutrino burst monitoring. Section 4 and 5 describe the second pipeline, focusing on the online reconstruction, the online neutrino classification and a preliminary event selection. Section 6 gives the first data-MC comparison based on the neutrino selection. Finally, Section 7 summarizes the work and gives the future outlook.

\subsection{The KM3NeT Detector}

Neutrinos can only be detected indirectly. When neutrinos interact in water, the secondary particles emit Cherenkov light. Detection and analysis of this Cherenkov light are used for the reconstruction of the primary neutrinos.

The KM3NeT infrastructure consists of building blocks, each building block is a 3D array of digital optical modules (DOMs) for recording the Cherenkov light. Each block has 115 vertical detection units (DUs), each DU with 18 DOMs. Each DOM has 31 3-inch PMTs. All the PMT analog signals passing a preset threshold are digitized and sent to shore via optical fibers. At the shore station, online data filtering is performed and the physics events are filtered from background. The arrival time and pulse amplitude observed by the PMTs are used to reconstruct the incoming neutrino's direction and energy.

The DU and DOM spacing are optimized for mainly two scientific goals, making up the two detectors of KM3NeT: ORCA (Oscillation Research with Cosmics in the Abyss) for the oscillation studies with atmospheric neutrinos in GeV scale, and ARCA (Astroparticle Research with Cosmics in the Abyss) for neutrino astronomy studies with astrophysical neutrinos up to a few PeV. ORCA will have one building block, with a vertical $9 \mathrm{~m}$ DOM spacing and a horizontal $20 \mathrm{~m}$ DU spacing, and ARCA two building blocks with a vertical $36 \mathrm{~m}$ DOM spacing and 90m DU spacing. The current KM3NeT detectors have six DUs of ARCA and finished Phase-1 of ORCA (six DUs since January 2020, referred to as ORCA6 from now on). 


\section{Real-time Alert Analysis Framework}

The KM3NeT real-time alert analysis framework has two pipelines: 1) the MeV CCSN monitoring pipeline, 2) the $\mathrm{GeV}-\mathrm{PeV}$ neutrino alert pipeline.

The MeV CCSN pipeline takes raw PMT data as input, using the coincidences of PMT hits inside each DOM. Each DOM acts as a standalone detector. The main goal of this pipeline is to provide early warning for optical telescopes for the observation of the next Galactic CCSN, as supernova neutrinos will arrive hours before the electromagnetic signals.

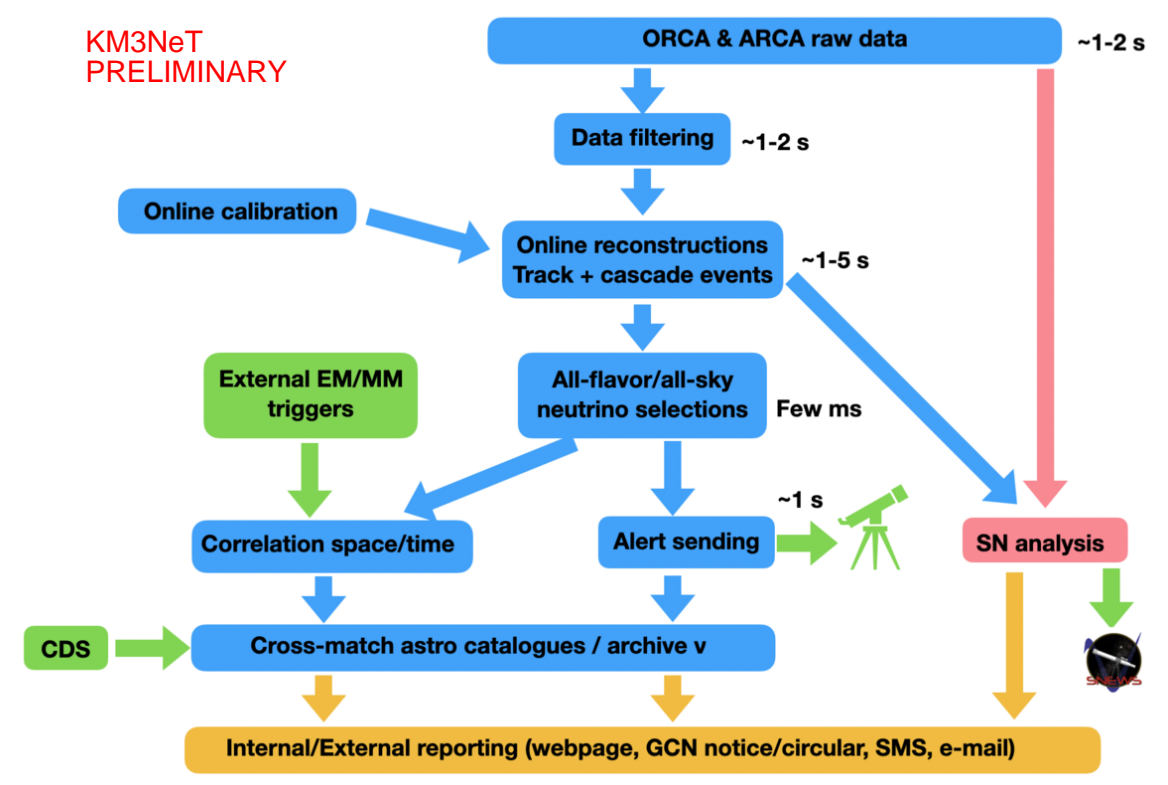

Figure 1: Data flow and the main structures of the KM3NeT real-time analysis framework. The blue boxes and arrows are for the GeV-PeV pipeline, the red box and arrow are for the CCSN pipeline. The green boxes show the alert receiver and the catalogues of the astronomical objects outside the solar system from CDS (Astronomical Data Center). The yellow box shows the internal or external reporting tools if there is a match after the correlation search.

The GeV-PeV pipeline uses the regular triggered events based on the coincidences of multiple DOMs. The goals of the real-time (GeV-PeV) neutrino alert pipeline are: 1) conduct online neutrino point source searches; 2) receive external electromagnetic, gravitational wave, or neutrino alerts and perform online neutrino correlation search based on these external alerts, 3) send all-flavor, all-sky neutrino real-time alerts to external observatories for follow-up. This entails a fast online reconstruction and a fast selection of a high-purity neutrino sample.

Fig. 1 shows the data flow and the main structures of the framework. The CCSN pipeline performs searches directly on the raw PMT data, the alerts are sent to SNEWS (SuperNova Early Warning System) [2,3]. The main processing steps of the GeV-PeV pipeline are represented by the blue boxes, where the raw PMT data are first filtered by the online data filtering [1] tool, then the physics events filtered out of the background undergo event processing which mainly include online event reconstruction and event classification. Based on the classification results, a highpurity event sample is selected for analysis. The analysis of the event sample will produce either 
interesting KM3NeT neutrino events (e.g., high energy or multiplets) suitable for issuing alerts (via the alert sender) or events correlated with external alerts that will be reported through multiple channels, mainly through GCN (The Gamma-ray Coordinates Network). The processing time is estimated for the main processing steps. In total, the framework's response time is on the order of 10 seconds. Currently, the combined elapsed time for online data filtering, track reconstruction, and classification is $4 \mathrm{~s}$ on average for ORCA6.

The event processing is conducted separately at the ORCA and ARCA shore station, but after the event classification, ORCA and ARCA data streams will be combined for analysis and any subsequent alerts.

\section{MeV Core-Collapse Supernova Neutrino Pipeline}

With the typical MeV-scale energy, one single supernova neutrino can't generate coincident light in multiple DOMs, but the large number of them (up to thousands) during a CCSN burst will result in higher counting rates of individual PMTs and an increase of the number of coincident hit PMTs within a DOM (termed multiplicity). Multiplicity is the main parameter that gives the signal/background separation.

Fig.2 (taken from [4]) shows the expected event rates from simulated CCSN bursts and the background rates as a function of multiplicity. The KM3NeT CCSN pipeline evaluates the multiplicity every $0.1 \mathrm{~s}$ in a $0.5 \mathrm{~s}$ sliding window. It is operational, sending alerts with false alarm rate less than 1/week, to SNEWS [2,3]. Alert generation latency is less than $20 \mathrm{~s}$.

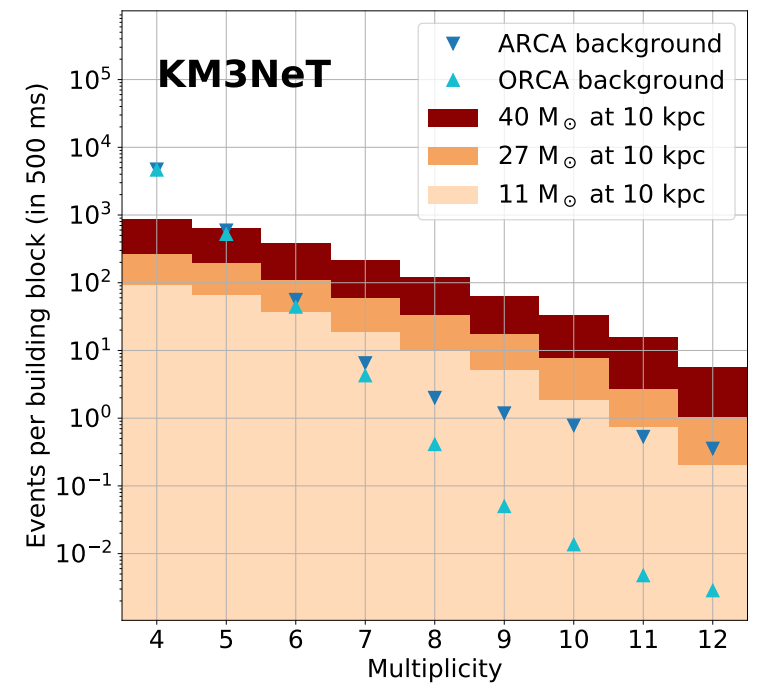

Figure 2: Supernova events expected from 3 simulated progenitors at ORCA and ARCA as a function of different multiplicity values compared with background rates (mainly from ${ }^{40} \mathrm{~K}$ decays and bioluminescence) [4].

\section{Online Reconstruction of the GeV-PeV Neutrino Pipeline}

Neutrinos in the GeV-PeV range can trigger multiple DOMs in the detector, they leave two main types of signatures: 1) track-like events with a visible track, mainly from the charged-current 
(CC) interactions of muon neutrinos and partially from the CC interactions of tau neutrinos; 2) shower-like events that have no visible muon track, these events come from the electron neutrino $\mathrm{CC}$ interactions, the neutral-current $(\mathrm{NC})$ interactions of all neutrino flavors and the majority of tau neutrino $\mathrm{CC}$ interactions.

Based on the two event signatures, KM3NeT event reconstruction can be divided into two types: track reconstruction [5] and shower reconstruction [6]. Online reconstruction uses the same algorithms as in offline, although it does not run the complete reconstruction chain for every event. Optimizations are under way, for example the shower reconstruction may be skipped for events that are clearly down-going atmospheric muons for efficiency reasons.

The muon track of the track-like events gives good directional resolution. Fig.3 shows the angular resolutions of ORCA $6 v_{\mu} \mathrm{CC}$ events, comparing the reconstruction level and a preliminary selection level (see next section). The selection gives a resolution close to the kinematic limit, at $9^{\circ}$ around $10 \mathrm{GeV}$ and up to $1^{\circ}$ at $\mathrm{TeV}$ scale. The angular resolution is limited by the size of the detector and should improve as more DUs get implemented.

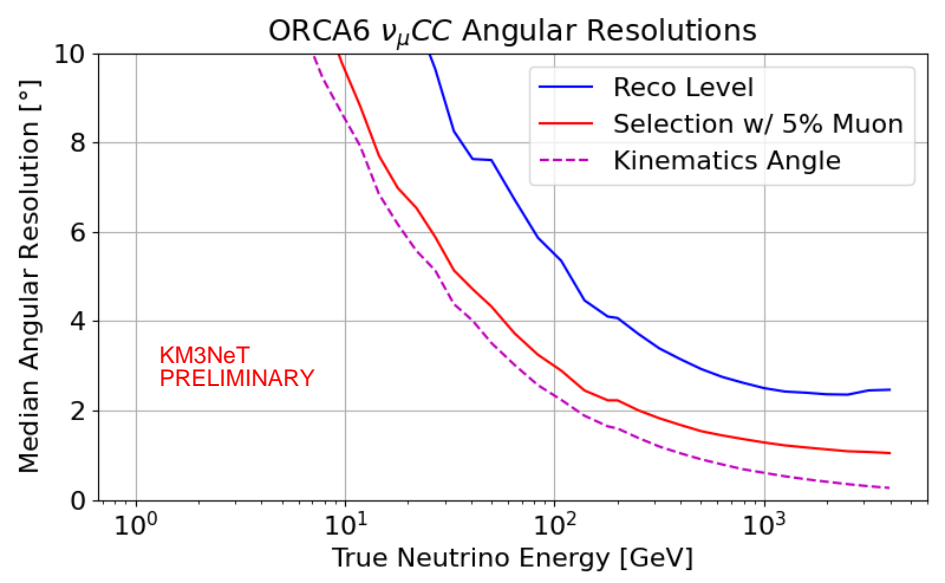

Figure 3: Angular resolution comparisons for preliminary selection and at the reconstruction level with ORCA6, the dashed line is the kinematic angle between the true neutrino and true muon. With the selection, the angular resolution can reach the order of $1^{\circ}$ at $\mathrm{TeV}$ range.

The online reconstruction is fast: the track direction reconstruction for ORCA6 takes $0.3 \mathrm{~s}$ per event on average, the shower reconstruction $1 \mathrm{~s}$. Events with more hits take a longer time, also events with more DUs will take longer, but overall the reconstruction time will stay on the order of $10 \mathrm{~s}$.

\section{Online Event Classification}

After events are reconstructed, they will undergo classification that selects neutrinos out of the enormous background of atmospheric muons. Each event is evaluated with a classification score indicating the probability of it being a neutrino. The processing of each event takes $0.01 \mathrm{~s}$.

The online classification is based on a classifier trained with a gradient boosting decision tree algorithm [7] with simulated $v_{\mu} \mathrm{CC}$ events as signals and atmospheric muons as backgrounds. The training features are physics parameters that provide good separation of neutrinos and atmospheric 
muons, for example the direction of the reconstructed muon track, the ratio of the track reconstruction's likelihood and number of degrees of freedom, the reconstructed neutrino interaction vertex position, the sum of ToT (Time Over Threshold, proxy for the charge observed by the triggered DOMs), and other geometry, charge and time-based parameters.

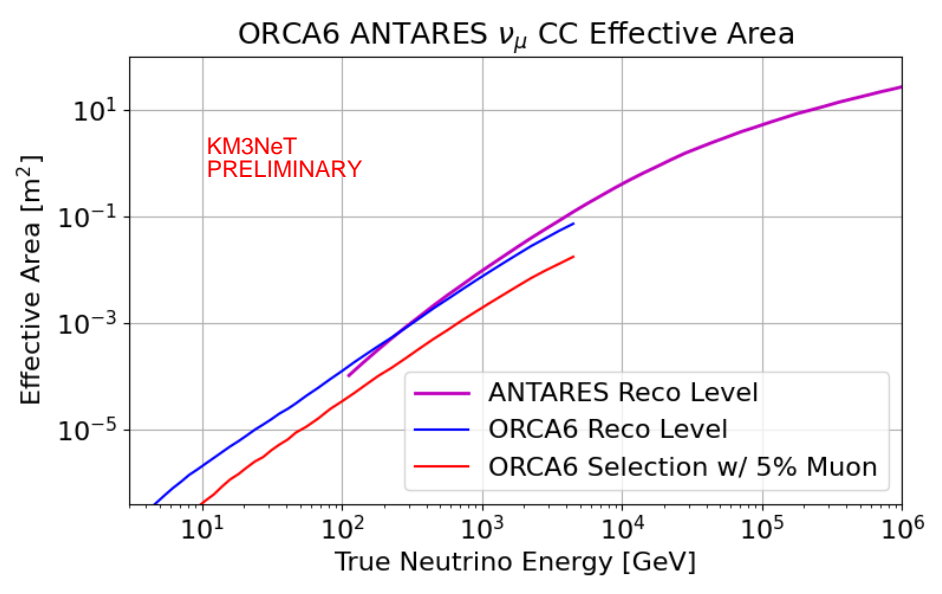

Figure 4: Effective area comparison for ANTARES, ORCA6 at reconstruction level, and the ORCA6 preliminary selection, which selects events uniformly across the energy range.

After the classification, a neutrino sample can be selected - the selection is about choosing the appropriate cut on the classification scores, and the cut can be different depending on different analyses. Fig. 3 and Fig. 4 show the resolution and effective area with a preliminary selection that gives 5\% muon contamination rate. The selection includes a sanity cut (on the triggered hits charge to remove events with sparking DOMs), a cut on the triggered number of hits $(>=20)$ and triggered number of lines $(>=2)$ to remove noise, a cut on the classification score $(>=0.9996)$, and finally a cut to select up-going events (reconstructed cos(zenith) $<0.1$ ). The noise cut is not optimized and thus may be too strict. A full noise simulation is also planned to be added in the classifier training. With this selection, the background muon rate is reduced by $10^{6}$ times while keeping $19 \%$ of all-sky signal atmospheric muon neutrinos (38\% of the up-going neutrinos), giving roughly 10 atmospheric neutrinos (among them $8.4 v_{\mu}$ neutrinos) and 0.5 background atmospheric muons per day in ORCA6. The training of shower classifiers and optimizations for transient source searches are underway.

\section{Event Selection Applied on Data}

This selection has been applied to the first year of ORCA6 dataset (341 days of livetime). This muon neutrino selection has been optimized to reduce the atmospheric muon contamination to $<5 \%$. Neutrino events are weighted with atmospheric fluxes [8] and oscillation parameters with NuFIT5.0 [9]; normal hierarchy is used. No systematics are considered here. On the first year of ORCA6 data, this selects 2879 up-going muon neutrino events. Fig. 5 shows the distributions of six parameters: the classification score, the ratio of the track reconstruction likelihood and number of degrees of freedom ( the most important training feature for the classifier), reconstructed cos(zenith), reconstructed energy, reconstructed zenith, and the sum of ToT. This first test on data gives a decent 

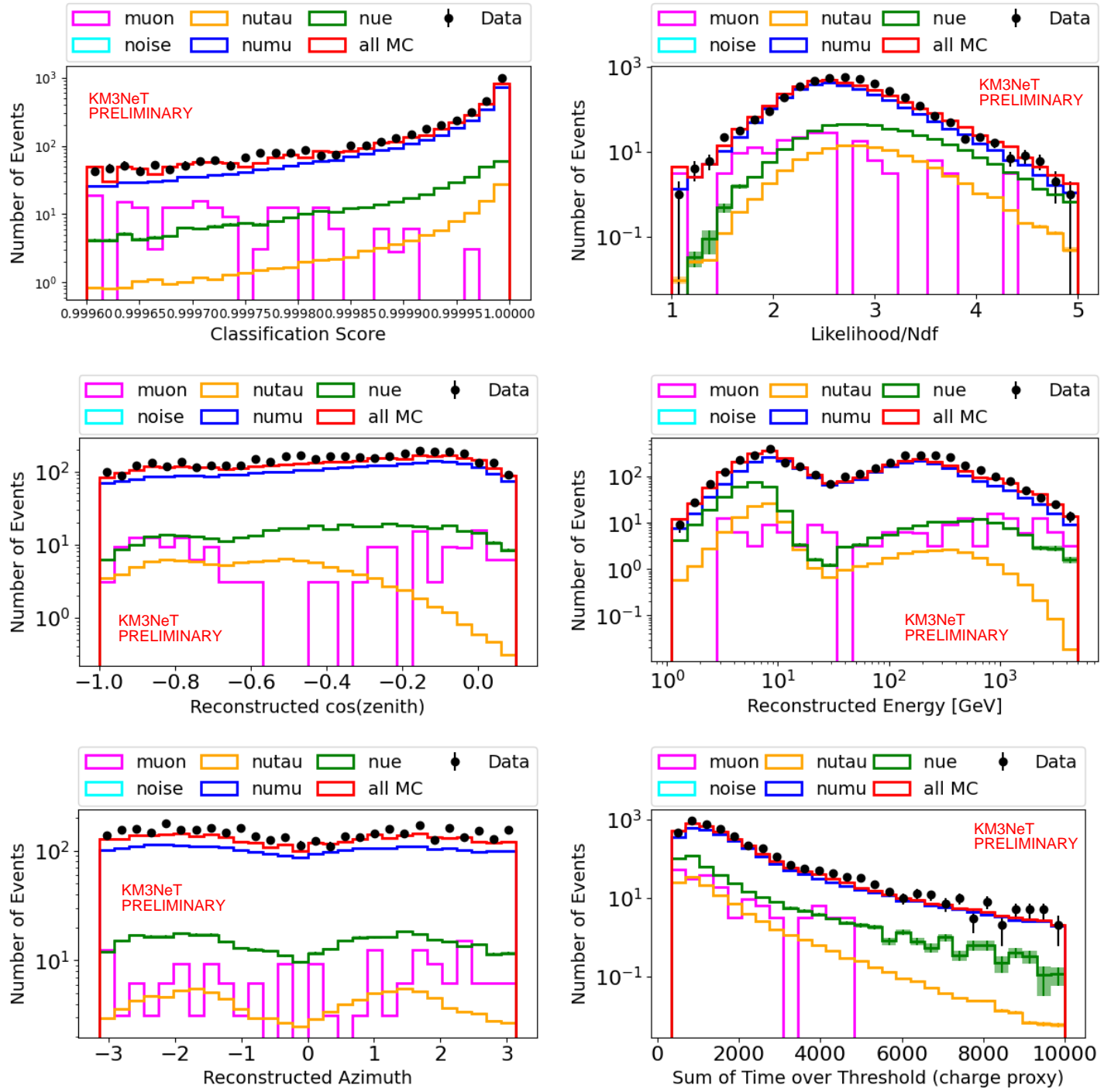

Figure 5: Data/MC comparison of the selected events. Upper left: classification score distribution. Upper right: ratio of track reconstruction likelihood and number of degrees of freedom. Middle left: reconstructed cosine(zenith). Middle right: reconstructed energy. Lower left: reconstructed azimuth. Lower right: sum of ToT of triggered hits. Data is shown in black dots. The blue histogram is for the muon MC (CC and NC combined), green for electron $\mathrm{CC}$ neutrinos, yellow for tau neutrino $\mathrm{CC}$, magenta for atmospheric muons, cyan for noise (note all noise are cleaned after the selection, so no cyan lines are actually present), and red histogram is the sum of all the MC events above.

data/MC agreement with the preliminary neutrino selection. In the future, more refinement can be considered taking into account each transient/variable analyses.

\section{Summary and Outlook}

The excellent angular resolution and large sky coverage of KM3NeT means KM3NeT will become a key contributor to the neutrino multi-messenger community. The current operational 
six lines of ORCA can already achieve a similar effective area to ANTARES. Our real-time multimessenger framework is under active development, and we expect to send out KM3NeT multimessenger alerts beginning in 2022. The alerts will be sent privately during the commissioning phase. After the full validation of the alert system, the main KM3NeT alerts will be distributed publicly.

\section{References}

[1] S Adrián-Martínez, et al. (KM3NeT Coll.), Journal of Physics G: Nuclear and Particle Physics, 43(8):084001 (2016)

[2] P. Antonioli, et al., SNEWS: The Supernova Early Warning System. New J. Phys. 6 pp. 114 (2004)

[3] S. Al Kharusi, et al., SNEWS 2.0: A next-generation supernova early warning system for multi-messenger astronomy. New J. Phys.. 23, 031201 (2021)

[4] S. Aiello, et al. (KM3NeT Coll.), The KM3NeT potential for the next core-collapse supernova observation with neutrinos. Eur. Phys. J. C. 81, 445 (2021)

[5] K. Melis, A. Heijboer, M. De Jong, (KM3NeT Coll.), KM3NeT/ARCA Event Reconstruction Algorithms, PoS (ICRC2017) 950 (2018)

[6] A. Domi, Shower Reconstruction and Sterile Neutrino Analysis with KM3NET/ORCA and ANTARES. (PhD Thesis) http://hdl.handle.net/11567/985989 (2019)

[7] T. Chen, \& C. Guestrin, XGBoost: A Scalable Tree Boosting System. Proceedings Of The 22nd ACM SIGKDD International Conference On Knowledge Discovery And Data Mining. pp. 785-794 (2016), http://doi.acm.org/10.1145/2939672.2939785

[8] Honda, M., Athar, M., Kajita, T., Kasahara, K. \& Midorikawa, S. Atmospheric neutrino flux calculation using the NRLMSISE-00 atmospheric model. Phys. Rev. D. 92, 023004 (2015,7)

[9] Esteban, I., Gonzalez-Garcia, M., Maltoni, M., Schwetz, T. \& Zhou, A. The fate of hints: updated global analysis of three-flavor neutrino oscillations. JHEP. 9 pp. 178 (2020), NuFIT 5.0 (2020), www.nu-fit.org. 


\section{Full Author List: KM3NeT Collaboration}

M. Ageron ${ }^{1}$, S. Aiello ${ }^{2}$, A. Albert ${ }^{3,55}$, M. Alshamsi ${ }^{4}$, S. Alves Garre ${ }^{5}$, Z. Aly ${ }^{1}$, A. Ambrosone ${ }^{6,7}$, F. Ameli ${ }^{8}$, M. Andre 9 , G. Androulakis ${ }^{10}$, M. Anghinolfi ${ }^{11}$, M. Anguita ${ }^{12}$, G. Anton ${ }^{13}$, M. Ardid $^{14}$, S. Ardid $^{14}$, W. Assal ${ }^{1}$, J. Aublin ${ }^{4}$, C. Bagatelas ${ }^{10}$, B. Baret ${ }^{4}$, S. Basegmez du Pree ${ }^{15}$, M. Bendahman ${ }^{4,16}$, F. Benfenati ${ }^{17,18}$, E. Berbee ${ }^{15}$, A. M. van den Berg ${ }^{19}$, V. Bertin ${ }^{1}$, S. Beurthey ${ }^{1}$, V. van Beveren ${ }^{15}$, S. Biagi ${ }^{20}$, M. Billault ${ }^{1}$, M. Bissinger ${ }^{13}$, M. Boettcher ${ }^{21}$, M. Bou Cabo ${ }^{22}$, J. Boumaaza ${ }^{16}$, M. Bouta ${ }^{23}$, C. Boutonnet ${ }^{4}$, G. Bouvet ${ }^{24}$, M. Bouwhuis ${ }^{15}$, C. Bozza ${ }^{25}$, H.Brânzass ${ }^{26}$, R. Bruijn ${ }^{15,27}$, J. Brunner ${ }^{1}$, R. Bruno ${ }^{2}$, E. Buis ${ }^{28}$, R. Buompane ${ }^{6,29}$, J. Busto ${ }^{1}$, B. Caiffi ${ }^{11}$, L. Caillat ${ }^{1}$, D. $\mathrm{Calvo}^{5}$, S. Campion ${ }^{30,8}$, A. Capone ${ }^{30,8}$, H. Carduner ${ }^{24}$, V. Carretero ${ }^{5}$, P. Castaldi ${ }^{17,31}$, S. Celli ${ }^{30,8}$, R. Cereseto ${ }^{11}$, M. Chabab ${ }^{32}$, C. Champion ${ }^{4}$, N. $\mathrm{Chau}^{4}$, A. Chen ${ }^{33}$, S. Cherubinini ${ }^{20,34}$, V. Chiarella ${ }^{35}$, T. Chiarusi ${ }^{17}$, M. Circella ${ }^{36}$, R. Cocimano ${ }^{20}$, J. A. B. Coelho ${ }^{4}$, A. Coleiro ${ }^{4}$, M. Colomer Molla ${ }^{4,5}$, S. Colonges ${ }^{4}$, R. Coniglione ${ }^{20}$, A. Cosquer ${ }^{1}$, P. Coyle ${ }^{1}$, M. Cresta ${ }^{11}$, A. Creusot ${ }^{4}$, A. $\mathrm{Cruz}^{37}$, G. Cuttone ${ }^{20}$, A. D’Amico ${ }^{15}$, R. Dallier ${ }^{24}$, B. De Martino ${ }^{1}$, M. De Palma ${ }^{36,38}$, I. Di Palma ${ }^{30,8}$, A. F. Díaz ${ }^{12}$, D. Diego$\operatorname{Tortosa}^{14}$, C. Distefano ${ }^{20}$, A. Domi ${ }^{15,27}$, C. Donzaud ${ }^{4}$, D. Dornic ${ }^{1}$, M. Dörr ${ }^{39}$, D. Drouhin ${ }^{3,55}$, T. Eberl ${ }^{13}$, A. Eddyamoui ${ }^{16}$, T. van Eeden ${ }^{15}$, D. van Eijk ${ }^{15}$, I. El Bojaddaini ${ }^{23}$, H. Eljarrari ${ }^{16}$, D. Elsaesser ${ }^{39}$, A. Enzenhöfer ${ }^{1}$, V. Espinosa ${ }^{14}$, P. Fermani ${ }^{30,8}$, G. Ferrara ${ }^{20,34}$, M. D. Filipović ${ }^{40}$, F. Filippini ${ }^{17,18}$, J. Fransen ${ }^{15}$, L. A. Fusco ${ }^{1}$, D. Gajanana ${ }^{15}$, T. Gal ${ }^{13}$, J. García Méndez ${ }^{14}$, A. Garcia Soto $^{5}$, E. Garçon ${ }^{1}$, F. Garufi ${ }^{6,7}$, C. Gatius ${ }^{15}$, N. Geißelbrecht ${ }^{13}$, L. Gialanella ${ }^{6,29}$, E. Giorgio ${ }^{20}$, S. R. Gozzini ${ }^{5}$, R. Gracia ${ }^{15}$, K. Graf ${ }^{13}$, G. Grella ${ }^{41}$, D. Guderian ${ }^{56}$, C. Guidi ${ }^{11,42}$, B. Guillon ${ }^{43}$, M. Gutiérrez ${ }^{44}$, J. Haefner ${ }^{13}$, S. Hallmann ${ }^{13}$, H. Hamdaoui ${ }^{16}$, H. van Haren ${ }^{45}$, A. Heijboer ${ }^{15}$, A. Hekalo ${ }^{39}$, L. Hennig ${ }^{13}$, S. Henry ${ }^{1}$, J. J. Hernández-Rey ${ }^{5}$, J. Hofestädt ${ }^{13}$, F. Huang ${ }^{1}$, W. Idrissi Ibnsalih ${ }^{6,29}$, A. Ilioni ${ }^{4}$, G. Illuminati ${ }^{17,18,4}$, C. W. James ${ }^{37}$, D. Janezashvili ${ }^{46}$, P. Jansweijer ${ }^{15}$, M. de Jong ${ }^{15,47}$, P. de Jong ${ }^{15,27}$, B. J. Jung ${ }^{15}$, M. Kadler ${ }^{39}$, P. Kalaczyński ${ }^{48}$, O. Kalekin ${ }^{13}$, U. F. Katz ${ }^{13}$, F. Kayzel $^{15}$, P. Keller ${ }^{1}$, N. R. Khan Chowdhury ${ }^{5}$, G. Kistauri ${ }^{46}$, F. van der Knaap ${ }^{28}$, P. Kooijman ${ }^{27,57}$, A. Kouchner ${ }^{4,49}$, M. Kreter ${ }^{21}$, V. Kulikovskiy ${ }^{11}$, M. Labalme ${ }^{43}$, P. Lagier ${ }^{1}$, R. Lahmann ${ }^{13}$, P. Lamare ${ }^{1}$, M. Lamoureux ${ }^{14}$, G. Larosa ${ }^{20}$, C. Lastoria ${ }^{1}$, J. Laurence ${ }^{1}$, A. Lazo $^{5}$, R. Le Breton ${ }^{4}$, E. Le Guirriec ${ }^{1}$, S. Le Stum ${ }^{1}$, G. Lehaut ${ }^{43}$, O. Leonardi ${ }^{20}$, F. Leone ${ }^{20,34}$, E. Leonora ${ }^{2}$, C. Lerouvillois ${ }^{1}$, J. Lesrel ${ }^{4}$, N. Lessing ${ }^{13}$, G. Levi ${ }^{17,18}$, M. Lincetto ${ }^{1}$, M. Lindsey Clark ${ }^{4}$, T. Lipreau ${ }^{24}$, C. LLorens Alvarez ${ }^{14}$, A. Lonardo ${ }^{8}$, F. Longhitano ${ }^{2}$, D. Lopez-Coto ${ }^{44}$, N. Lumb ${ }^{1}$, L. Maderer ${ }^{4}$, J. Majumdar ${ }^{15}$, J. Mańczak ${ }^{5}$, A. Margiotta ${ }^{17,18}$, A. Marinelli ${ }^{6}$, A. Marini ${ }^{1}$, C. Markou $^{10}$, L. Martin ${ }^{24}$, J. A. Martínez-Mora ${ }^{14}$, A. Martini ${ }^{35}$, F. Marzaioli ${ }^{6,29}$,

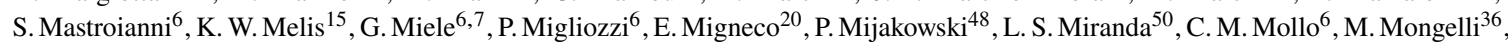
A. Moussa ${ }^{23}$, R. Muller ${ }^{15}$, P. Musico ${ }^{11}$, M. Musumeci ${ }^{20}$, L. Nauta ${ }^{15}$, S. Navas ${ }^{44}$, C. A. Nicolau ${ }^{8}$, B. Nkosi ${ }^{33}$, B. Ó Fearraigh ${ }^{15,27}$, M. O'Sullivan ${ }^{37}$, A. Orlando ${ }^{20}$, G. Ottonello ${ }^{11}$, S. Ottonello ${ }^{11}$, J. Palacios González ${ }^{5}$, G. Papalashviliite, R. Papaleo ${ }^{20}$, C. Pastore ${ }^{36}$, A. M. Păun ${ }^{26}$, G.E. Păvălaşş, G. Pellegrinini ${ }^{17}$, C. Pellegrino ${ }^{18,58}$, M. Perrin-Terrinin ${ }^{1}$, V. Pestel ${ }^{15}$, P. Piattelli ${ }^{20}$, C. Pieterse ${ }^{5}$, O. Pisanti ${ }^{6,7}$, C. Poirè ${ }^{14}$, V. Popa $^{26}$, T. Pradier $^{3}$, F. Pratolongo ${ }^{11}$, I. Probst ${ }^{13}$, G. Pühlhofer ${ }^{51}$, S. Pulvirenti ${ }^{20}$, G. Quéméner ${ }^{43}$, N. Randazzo ${ }^{2}$, A. Rapicavoli ${ }^{34}$, S. Razzaque $^{50}$, D. Real ${ }^{5}$, S. Reck ${ }^{13}$, G. Riccobene ${ }^{20}$, L. Rigalleau ${ }^{24}$, A. Romanov ${ }^{11,42}$, A. Rovelli ${ }^{20}$, J. Royon ${ }^{1}$, F. Salesa Greus ${ }^{5}$, D. F. E. Samtleben ${ }^{15,47}$, A. Sánchez Losa ${ }^{36,5}$, M. Sanguineti ${ }^{11,42}$, A. Santangelo ${ }^{51}$, D. Santonocito ${ }^{20}$, P. Sapienza ${ }^{20}$, J. Schmelling ${ }^{15}$, J. Schnabel ${ }^{13}$, M. F. Schneider ${ }^{13}$, J. Schumann ${ }^{13}$, H. M. Schutte ${ }^{21}$, J. Seneca ${ }^{15}$, I. Sgura ${ }^{36}$, R. Shanidze ${ }^{46}$, A. Sharma ${ }^{52}$, A. Sinopoulou ${ }^{10}$, B. Spisso ${ }^{41,6}$, M. Spurio ${ }^{17,18}$, D. Stavropoulos ${ }^{10}$, J. Steijger ${ }^{15}$, S. M. Stellacci ${ }^{41,6}$, M. Taiuti ${ }^{11,42}$, F. Tatone ${ }^{36}$, Y. Tayalati ${ }^{16}$, E. Tenllado ${ }^{44}$, D. Tézier ${ }^{1}$, T. Thakore ${ }^{5}$, S. Theraube ${ }^{1}$, H. Thiersen $^{21}$, P. Timmer $^{15}$, S. Tingay ${ }^{37}$, S. Tsagkli $^{10}$, V. Tsourapis ${ }^{10}$, E. Tzamariudaki ${ }^{10}$, D. Tzanetatos ${ }^{10}$, C. Valieri ${ }^{17}$, V. Van Elewyck ${ }^{4,49}$, G. Vasileiadis ${ }^{53}$, F. Versari ${ }^{17,18}$, S. Viola $^{20}$, D. Vivolo ${ }^{6,29}$, G. de Wasseige ${ }^{4}$, J. Wilms ${ }^{54}$, R. Wojaczyński ${ }^{48}$, E. de Wolf ${ }^{15,27}$, T. Yousfi ${ }^{23}$, S. Zavatarelli ${ }^{11}$, A. Zegarelli ${ }^{30,8}$, D. Zito ${ }^{20}$, J. D. Zornoza ${ }^{5}$, J. Zúñiga ${ }^{5}$, N. Zywucka ${ }^{21}$.

${ }^{1}$ Aix Marseille Univ, CNRS/IN2P3, CPPM, Marseille, France.

${ }^{2}$ INFN, Sezione di Catania, Via Santa Sofia 64, Catania, 95123 Italy.

${ }^{3}$ Université de Strasbourg, CNRS, IPHC UMR 7178, F-67000 Strasbourg, France.

${ }^{4}$ Université de Paris, CNRS, Astroparticule et Cosmologie, F-75013 Paris, France.

${ }^{5}$ IFIC - Instituto de Física Corpuscular (CSIC - Universitat de València), c/Catedrático José Beltrán, 2, 46980 Paterna, Valencia, Spain. ${ }^{6}$ INFN, Sezione di Napoli, Complesso Universitario di Monte S. Angelo, Via Cintia ed. G, Napoli, 80126 Italy.

${ }^{7}$ Università di Napoli “Federico II", Dip. Scienze Fisiche "E. Pancini”, Complesso Universitario di Monte S. Angelo, Via Cintia ed. G, Napoli, 80126 Italy.

${ }^{8}$ INFN, Sezione di Roma, Piazzale Aldo Moro 2, Roma, 00185 Italy.

${ }^{9}$ Universitat Politècnica de Catalunya, Laboratori d'Aplicacions Bioacústiques, Centre Tecnològic de Vilanova i la Geltrú, Avda. Rambla Exposició, s/n, Vilanova i la Geltrú, 08800 Spain.

${ }^{10}$ NCSR Demokritos, Institute of Nuclear and Particle Physics, Ag. Paraskevi Attikis, Athens, 15310 Greece.

${ }^{11}$ INFN, Sezione di Genova, Via Dodecaneso 33, Genova, 16146 Italy.

${ }^{12}$ University of Granada, Dept. of Computer Architecture and Technology/CITIC, 18071 Granada, Spain.

${ }^{13}$ Friedrich-Alexander-Universität Erlangen-Nürnberg, Erlangen Centre for Astroparticle Physics, Erwin-Rommel-Straße 1, 91058 Erlangen, Germany.

${ }^{14}$ Universitat Politècnica de València, Instituto de Investigación para la Gestión Integrada de las Zonas Costeras, C/ Paranimf, 1, Gandia, 46730 Spain.

${ }^{15}$ Nikhef, National Institute for Subatomic Physics, PO Box 41882, Amsterdam, 1009 DB Netherlands.

${ }^{16}$ University Mohammed V in Rabat, Faculty of Sciences, 4 av. Ibn Battouta, B.P. 1014, R.P. 10000 Rabat, Morocco.

${ }^{17}$ INFN, Sezione di Bologna, v.le C. Berti-Pichat, 6/2, Bologna, 40127 Italy.

\footnotetext{
${ }^{1}$ also at Dipartimento di Fisica, INFN Sezione di Padova and Università di Padova, I-35131, Padova, Italy
} 
${ }^{18}$ Università di Bologna, Dipartimento di Fisica e Astronomia, v.le C. Berti-Pichat, 6/2, Bologna, 40127 Italy.

${ }^{19}$ KVI-CART University of Groningen, Groningen, the Netherlands.

${ }^{20}$ INFN, Laboratori Nazionali del Sud, Via S. Sofia 62, Catania, 95123 Italy.

${ }^{21}$ North-West University, Centre for Space Research, Private Bag X6001, Potchefstroom, 2520 South Africa.

${ }^{22}$ Instituto Español de Oceanografía, Unidad Mixta IEO-UPV, C/ Paranimf, 1, Gandia, 46730 Spain.

${ }^{23}$ University Mohammed I, Faculty of Sciences, BV Mohammed VI, B.P. 717, R.P. 60000 Oujda, Morocco.

${ }^{24}$ Subatech, IMT Atlantique, IN2P3-CNRS, Université de Nantes, 4 rue Alfred Kastler - La Chantrerie, Nantes, BP 2072244307 France.

${ }^{25}$ Università di Salerno e INFN Gruppo Collegato di Salerno, Dipartimento di Matematica, Via Giovanni Paolo II 132, Fisciano, 84084 Italy.

${ }^{26}$ ISS, Atomistilor 409, Măgurele, RO-077125 Romania.

${ }^{27}$ University of Amsterdam, Institute of Physics/IHEF, PO Box 94216, Amsterdam, 1090 GE Netherlands.

${ }^{28}$ TNO, Technical Sciences, PO Box 155, Delft, 2600 AD Netherlands.

${ }^{29}$ Università degli Studi della Campania "Luigi Vanvitelli", Dipartimento di Matematica e Fisica, viale Lincoln 5, Caserta, 81100 Italy.

${ }^{30}$ Università La Sapienza, Dipartimento di Fisica, Piazzale Aldo Moro 2, Roma, 00185 Italy.

${ }^{31}$ Università di Bologna, Dipartimento di Ingegneria dell'Energia Elettrica e dell'Informazione "Guglielmo Marconi", Via dell’Università 50, Cesena, 47521 Italia.

${ }^{32}$ Cadi Ayyad University, Physics Department, Faculty of Science Semlalia, Av. My Abdellah, P.O.B. 2390, Marrakech, 40000 Morocco.

${ }^{33}$ University of the Witwatersrand, School of Physics, Private Bag 3, Johannesburg, Wits 2050 South Africa.

${ }^{34}$ Università di Catania, Dipartimento di Fisica e Astronomia "Ettore Majorana", Via Santa Sofia 64, Catania, 95123 Italy.

${ }^{35}$ INFN, LNF, Via Enrico Fermi, 40, Frascati, 00044 Italy.

${ }^{36}$ INFN, Sezione di Bari, via Orabona, 4, Bari, 70125 Italy.

${ }^{37}$ International Centre for Radio Astronomy Research, Curtin University, Bentley, WA 6102, Australia.

${ }^{38}$ University of Bari, Via Amendola 173, Bari, 70126 Italy.

${ }^{39}$ University Würzburg, Emil-Fischer-Straße 31, Würzburg, 97074 Germany.

${ }^{40}$ Western Sydney University, School of Computing, Engineering and Mathematics, Locked Bag 1797, Penrith, NSW 2751 Australia.

${ }^{41}$ Università di Salerno e INFN Gruppo Collegato di Salerno, Dipartimento di Fisica, Via Giovanni Paolo II 132, Fisciano, 84084 Italy.

${ }^{42}$ Università di Genova, Via Dodecaneso 33, Genova, 16146 Italy.

${ }^{43}$ Normandie Univ, ENSICAEN, UNICAEN, CNRS/IN2P3, LPC Caen, LPCCAEN, 6 boulevard Maréchal Juin, Caen, 14050 France.

${ }^{44}$ University of Granada, Dpto. de Física Teórica y del Cosmos \& C.A.F.P.E., 18071 Granada, Spain.

${ }^{45}$ NIOZ (Royal Netherlands Institute for Sea Research), PO Box 59, Den Burg, Texel, 1790 AB, the Netherlands.

${ }^{46}$ Tbilisi State University, Department of Physics, 3, Chavchavadze Ave., Tbilisi, 0179 Georgia.

${ }^{47}$ Leiden University, Leiden Institute of Physics, PO Box 9504, Leiden, 2300 RA Netherlands.

${ }^{48}$ National Centre for Nuclear Research, 02-093 Warsaw, Poland.

${ }^{49}$ Institut Universitaire de France, 1 rue Descartes, Paris, 75005 France.

${ }^{50}$ University of Johannesburg, Department Physics, PO Box 524, Auckland Park, 2006 South Africa.

${ }^{51}$ Eberhard Karls Universität Tübingen, Institut für Astronomie und Astrophysik, Sand 1, Tübingen, 72076 Germany.

${ }^{52}$ Università di Pisa, Dipartimento di Fisica, Largo Bruno Pontecorvo 3, Pisa, 56127 Italy.

${ }^{53}$ Laboratoire Univers et Particules de Montpellier, Place Eugène Bataillon - CC 72, Montpellier Cédex 05, 34095 France.

${ }^{54}$ Friedrich-Alexander-Universität Erlangen-Nürnberg, Remeis Sternwarte, Sternwartstraße 7, 96049 Bamberg, Germany.

${ }^{55}$ Université de Haute Alsace, 68100 Mulhouse Cedex, France.

${ }^{56}$ University of Münster, Institut für Kernphysik, Wilhelm-Klemm-Str. 9, Münster, 48149 Germany.

${ }^{57}$ Utrecht University, Department of Physics and Astronomy, PO Box 80000, Utrecht, 3508 TA Netherlands.

${ }^{58}$ INFN, CNAF, v.le C. Berti-Pichat, 6/2, Bologna, 40127 Italy. 